\title{
i-Co' $\mathbf{Q}$ : protocolo inteligente para el control de la congestión consciente de la QoS aplicado al monitoreo de signos vitales
}

\author{
Enriqueta Patricia Becerra-Sánchez, Antonio Guerrero-Ibáñez, \\ Raymundo Buenrostro-Mariscal \\ Universidad de Colima, Facultad de Telemática, Colima, \\ México \\ \{ebecerra0, antonio_guerrero, raymundo\}@ucol.mx
}

\begin{abstract}
Resumen. En los últimos años el uso de las redes de sensores enfocados al desarrollo de aplicaciones médicas se han incrementado especialmente para el monitoreo de signos vitales en pacientes, dando apertura al desarrollo de redes de área corporal (WBAN). Sin embargo, la información recolectada de las WBAN tiene diferentes requerimientos de comunicación (datos heterogéneos, fiabilidad de entrega, retardo, etc.). Las características naturales de operación de las WBAN hacen que sufran problemas de congestión, debido a la saturación del canal de comunicación y al desbordamiento de buffer provocando colisiones y desbordamiento que se traducen en pérdida de paquetes. Es así como surge i$\mathrm{Co}^{2} \mathrm{Q}$ un protocolo inteligente consciente de la calidad de servicio para el control de la congestión aplicado al monitoreo de signos vitales. Los resultados obtenidos muestran la eficiencia del protocolo propuesto en ambientes de la salud.
\end{abstract}

Palabras clave: WBAN, control de congestión, calidad de servicio (QoS).

\section{Introducción}

En los últimos años los avances tecnológicos han permitido el desarrollo de mejores servicios para el cuidado de la salud, proporcionando herramientas para el monitoreo de los signos vitales de los pacientes en tiempo real que apoyen los diagnósticos médicos [1], surgiendo el concepto de Redes de área corporal (WBANs por sus siglas en inglés Wireless Body Area Networks) las cuales están pensadas en el monitoreo remoto de los pacientes [2]. Estas redes se componen de un conjunto de sensores pequeños localizados dentro o fuera del cuerpo humano encargados de recolectar diferentes signos vitales (tales como la presión sanguínea, pulso, temperatura, electrocardiograma (ECG), entre otros) y transmitirlos a un dispositivo cercano llamado nodo Sink $[3,4]$.

Las WBANs generan datos heterogéneos, es decir, distintos tipos de datos y volúmenes de tráfico que a su vez tienen diferentes requerimientos de transmisión (por ejemplo velocidad de datos, confiabilidad y prioridad en la entrega), saturando la red de tráfico diverso, provocando colisiones, originando congestión, pérdida de paquetes y por consecuencia una baja calidad de servicio de comunicación[5][6]. 
Si se desea realizar un monitoreo de pacientes en tiempo real de forma eficiente, es necesario implementar protocolos que soporten calidad de servicio para mejorar la eficiencia de transmisión de la información dentro de un entorno, por ejemplo diferenciar los distintos flujos de información, prioridad de datos y controlar la congestión.

Es así como surge i-Co ${ }^{2} \mathrm{Q}$, un protocolo inteligente para el control de la congestión que permita detectarla, notificarla y mitigarla mejorando la eficiencia de la transmisión de los datos consciente de la calidad de servicio en la red.

El resto del artículo está organizado de la siguiente forma, la sección 2 presenta una serie de trabajos relacionados al tópico de nuestra propuesta. Una descripción detallada del protocolo $\mathrm{i}-\mathrm{Co}^{2} \mathrm{Q}$ se presenta en la sección 3. En la sección 4 se muestra la simulación realizada. La sección 5 muestra un análisis de los resultados obtenidos. Finalmente cerramos este trabajo con las conclusiones.

\section{Trabajos relacionados}

Dentro de la literatura podemos encontrar varios trabajos relacionados sobre el control de congestión y fiabilidad de la transmisión de los datos dentro del área de redes de sensores. En [6] se analizan varios de ellos y a continuación se presentan las investigaciones que más relación tienen con el presente trabajo.

El protocolo STCP [7] detecta la congestión local, utiliza un grado de utilización del buffer en cada nodo intermedio involucrado en la transmisión de datos, envía una notificación de congestión de forma implícita, es decir, fijando un bit en el campo de notificación de congestión (CN) del encabezado del paquete de datos y del paquete Ack. Cada nodo de la red tiene la posibilidad de detectar la congestión y generar la notificación hacia el Sink, cuando un nodo recibe la notificación de congestión, éste direcciona los paquetes sucesivos del flujo de datos por una ruta diferente, siempre y cuando se tenga un algoritmo en la capa de red que permita este proceso, o disminuir la velocidad de transmisión de los nodos fuente.

Por otro lado en [8] se presenta TRCCIT un mecanismo para detectar la congestión en los nodos mediante una comparación de la tasa de arribo de paquetes, contra la tasa de envío, notificando de forma inmediata a los vecinos de la congestión mediante la activación de un bit en cada paquete que sale del nodo. TRCCIT resuelve la congestión de forma pro-activa, es decir, selecciona varias rutas para redirigir el tráfico de los nodos fuente. Sin embargo, esto provoca que los paquetes tarden en llegar a su destino, incrementando el tiempo de transmisión de los datos.

El protocolo CODA [9] utiliza el nivel de ocupación del buffer para determinar la carga actual y detectar con más precisión la congestión en cada receptor, para difundir el grado de congestión utiliza la notificación explícita hacia los nodos fuente, al recibir este mensaje los nodos reducen la tasa de envío y retransmiten el aviso a los nodos vecinos, esta forma es conocida como backpressure. Sin embargo, al hacer retransmisiones a los nodos vecinos se consume mucha energía.

En [10] se presenta CTCP un protocolo que determina la presencia de congestión a través de la verificación del umbral del buffer y la pérdida de paquetes. Este protocolo utiliza un mecanismo de reconocimiento de paquetes que distingue si las causas de la congestión son por desbordamiento del buffer o por error de la transmisión, el nodo que 
detecta la congestión utiliza la notificación explícita generando mensajes a todos los nodos vecinos para indicarles que no pueden recibir más paquetes. Para resolver la congestión utiliza un mecanismo que ajustas gradualmente la tasa de transmisión en lo nodos fuente evitando una reducción agresiva. Sin embargo al detener la recepción de paquetes en los nodos vecinos, produce la pérdida paquetes.

En los trabajos de investigación mencionados anteriormente sobre el control de congestión, podemos notar que ninguno considera las necesidades de las aplicaciones; por lo tanto el protocolo i-Co ${ }^{2} \mathrm{Q}$ se enfoca en el control de congestión consiente de la calidad de servicio en aplicaciones médicas.

\section{Propuesta del protocolo i-Co $\mathrm{Co}^{2} \mathrm{Q}$}

Nuestro esquema se basa en un escenario de red en un entorno hospitalario, el cual consiste en el desplazamiento de los pacientes dentro de la zona de cobertura. Los pacientes llevan una serie de sensores interconectados a un nodo inalámbrico que permite recolectar información sobre sus signos vitales y los envía a un nodo intermedio; éste sirve como puente de comunicación entre el paciente y la computadora final donde la información es analizada por el personal médico (enfermera o médicos), tal y como se muestra en la figura 1 .

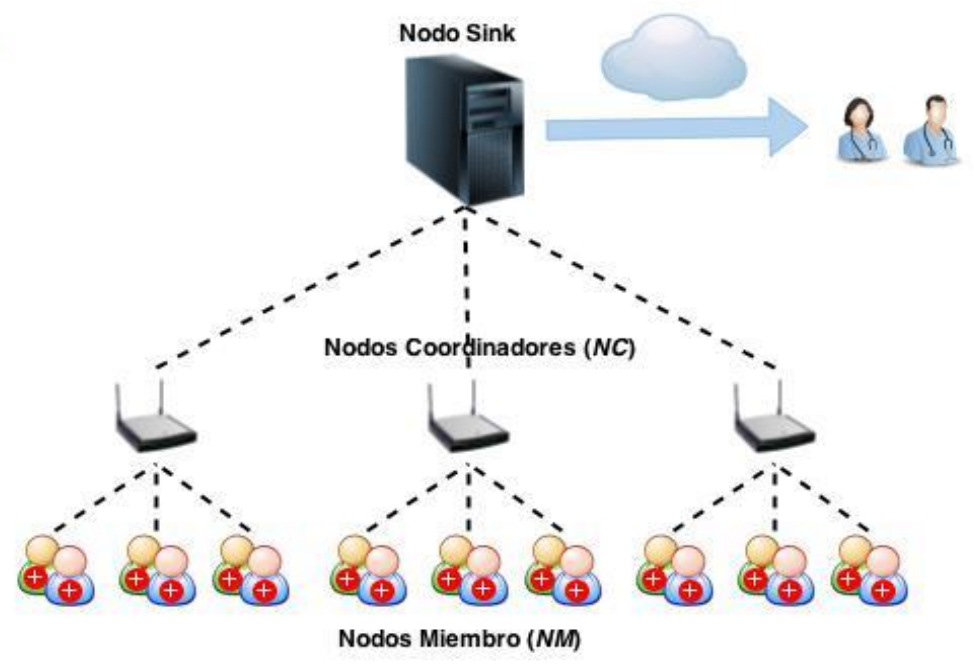

Fig. 1. Representación de la arquitectura física.

El esquema propuesto se dividió en dos arquitecturas: la física y la lógica. A continuación se describen sus módulos y componentes que conforman cada arquitectura.

\subsection{Arquitectura física}

La arquitectura física está compuesta de dispositivos interconectados en una red inalámbrica, basada en una estructura jerárquica de tres niveles conformada por 
pacientes en el nivel inferior, nodos coordinadores en el nivel intermedio y en el último nivel el nodo sink (Fig. 1).

El nivel uno formado por los nodos miembro (NM), representan los nodos compuestos con todos los sensores que trae interconectado cada uno de los pacientes, recolectando la información censada para después enviarla a los nodos en el nivel dos.

El segundo nivel de la estructura son los nodos coordinadores (NC) que tienen contacto directo con el nivel uno y tres, además de ser responsables de administrar una serie de NM.

Por último en el nivel tres se encuentra el equipo final denominado Sink (NS) donde se recibirán los datos censados, asimismo, este nivel es responsable de regular el registro y actualización de sesiones por paciente.

\subsection{Arquitectura lógica}

La arquitectura lógica de esta propuesta la conforman cuatro módulos principales: control de sesiones, recolectado y congestión los cuales son distribuidos entre los diferentes nodos que conforman la arquitectura física (figura 2).

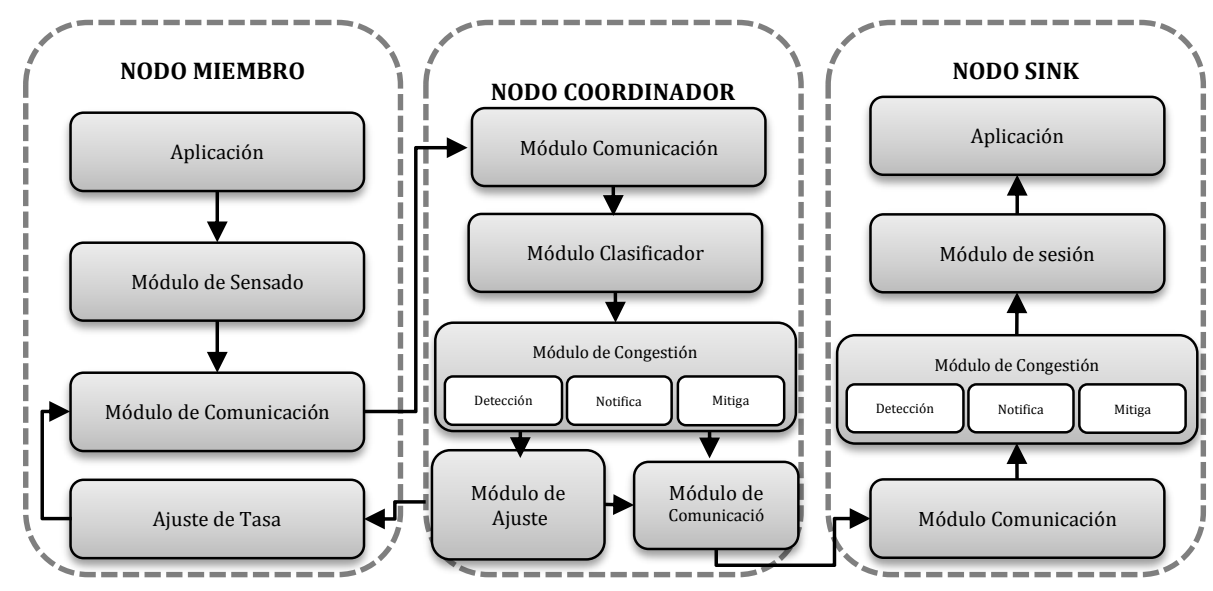

Fig. 2. Representación de la arquitectura lógica.

El módulo de sesión el cual se encuentra en el NS se encarga de registrar a cada NM en su tabla de gestión de sesión, indicando el NC al que está conectado de acuerdo a la estructura, además le asigna un número de sesión por nodo. Este proceso sólo se ejecuta cuando los nodos se activan por primera vez, cada NM envía un mensaje de broadcast para solicita su registro al NS, el cual creará una nueva sesión para el nuevo nodo.

El módulo de censado se encuentra localizado en el NM y es responsable de recolectar y enviar la información de cada nodo del paciente, con parámetros de frecuencia de envío y ancho de banda definidas dependiendo del tipo de información.

El módulo de comunicación situado en los tres nodos y es encargado de enviar y recibir los paquetes de manera fiable de extremo a extremo, cubriendo los requerimientos de las métricas tales como retardo de transmisión del paquete y la tasa de entrega de datos. 
El módulo clasificador se encuentra en NC y es encargado de identificar y clasificar los tipos de datos a través de su prioridad. La prioridad para cada tipo de dato la define el módulo de aplicación y se define con valores 1,2 y 3 donde 1 representa prioridad más alta. Este módulo implementa un sistema de colas permitiendo que los nodos intermedios cambien el orden de entrega de paquetes al nodo Sink, de acuerdo a su importancia (por ejemplo, en tiempo real, tiempo no real, tipo de aplicación, etc.). Este sistema permite reducir el retardo de extremo a extremo en la transmisión de datos, mejora la eficiencia en términos de tiempo de espera y mínimo consumo de energía.

El módulo de ajuste encargado de definir la nueva tasa de transmisión para cada uno de los nodos que conforman el NC donde se detecta la congestión. Para definir la nueva tasa de ajuste se considera la prioridad del tipo de datos y la perdida de paquetes del nodo donde se detectó la congestión. El ajuste se calcula para todo los nodos que tengan una prioridad menor o igual a la prioridad de dato (ec. 1).

$$
F_{\text {ajuste }}(i)=P_{i} N P P_{\text {Nodocong }} \text {, }
$$

donde $\mathrm{P}_{\mathrm{i}}$ representa la prioridad del tipo de dato y $\mathrm{NPP}_{\text {NodoCong }}$ es el número de paquetes perdidos del nodo congestionado.

El módulo de control de congestión ubicado en el NC y NS es responsable de medir la congestión de acuerdo a la pérdida de paquetes, controlando la tasa de flujo de paquetes y balanceo de carga, evitando retrasos o pérdida de paquetes y como consecuencia un bajo nivel de calidad de servicio. Este módulo se divide en tres fases esenciales las cuales se describen a continuación:

i) Detección de congestión: Esta fase permite detectar al NC o al NS la presencia de congestión dentro del área de cada nodo; para lograrlo utiliza un mecanismo de reconocimiento y control de la secuencia de paquetes transmitidos, este mecanismo se denomina incidencia y monitorea el número de secuencia de cada paquete recibido, si se detecta pérdida de paquetes se activa una incidencia, la finalidad de la incidencia es identificar situaciones tempranas de problemas de congestión.

ii) Notificación: cuando el NC detecta una violación en el porcentaje de paquetes perdidos o se rebasa el límite de incidencias definido, el NC propaga un mensaje de control de forma inmediata hacia los NM que lo conforman. Dentro del mensaje el NC tendrá que informar a los NM el reajuste adecuado de tasa de transmisión que se realiza en el módulo de ajuste de acuerdo al tipo de tráfico. La notificación de la congestión se hace de manera explícita, es decir, utiliza mensajes de notificación de congestión especiales para informar a los nodos implicados de la existencia de congestión.

iii) Mitigación: Para mitigar el problema de la congestión se toman acciones proactivas y dinámicas, permitiendo que cada NM de la red sea capaz de controlar su propio flujo de datos a través del ajuste de la tasa de tráfico dependiendo de las incidencias del NC. Utiliza el modo extremo a extremo (E2E - Por sus siglas en inglés end-to-end) que impone el ajuste de tasa exacta para cada NM. Si el NC sigue experimentando congestión, seguirá disminuyendo la tasa de flujo de datos hasta que se solvente el problema de congestión dentro del NC.

\section{Simulación de i-Co² $\mathrm{Q}$}

El desempeño de i-Co ${ }^{2} \mathrm{Q}$ se evaluó mediante simulaciones usando la herramienta CASTALIA [11] que está basada en Omnet++ [12]. El protocolo se comparó con un 
escenario donde no se aplica QoS (el cual denominamos no-QoS). Para la evaluación se analizaron las métricas de consumo de energía, porcentaje de paquetes recibidos por el Sink y paquetes perdidos.

\subsection{Escenario de la simulación}

Para la simulación se definió un área con dimensiones de 400 x 400m, formado con un total de 33 nodos: 1 nodo sink, 4 nodos coordinadores y 7 nodos miembro por cada cluster, los cuales fueron desplegados de manera determinística (los nodos se instalan manualmente y se les configuran rutas estáticas) [13], ubicando en el centro del campo el nodo sink, después los NC son ubicados alrededor del NS dentro de su cobertura y por último los NM conectados a los NC. Las configuraciones de los parámetros utilizados en la simulación se presentan a continuación en la tabla 1.

Tabla 1. Parámetros de la configuración.

\begin{tabular}{cc}
\hline Parámetros & Valores \\
\hline Tiempo de la simulación & $1000 \mathrm{~s}$ \\
Tasa de paquetes & 10 \\
Radio & $\mathrm{CC} 2420$ \\
Potencia de transmisión & $0 \mathrm{dBm}$ \\
Tipo de nodo & Estático \\
Protocolo de Enrutamiento & Enrutamiento estático \\
Protocolo MAC & TMAC \\
\hline
\end{tabular}

El modelo de colisión utilizado está basado en incrementar el impacto de varias señales de entrada en el nodo receptor. Dentro de la simulación se evaluaron tres valores de incidencia $(1,2$ y 3 ) con el objetivo de identificar cuál de estos valores permite obtener un mejor desempeño del protocolo propuesto.

\section{Resultados}

Para evaluar los resultados obtenidos del desempeño de $\mathrm{i}-\mathrm{Co}^{2} \mathrm{Q}$, se hizo la comparación con protocolos existentes dentro de la literatura que consideran entornos hospitalarios, a continuación se discuten dichos resultados.

La figura 3 a muestra los resultados obtenidos en relación a la cantidad de paquetes recibidos con éxito, al utilizar i-Co ${ }^{2} \mathrm{Q}$ el número total de paquetes recibidos por el nodo Sink es mayor teniendo un porcentaje por encima del $97 \%$, en comparación con el protocolo presentado en [3] que solo cuenta con el 94\% de la transmisión, también se hace una comparación al escenario donde no se aplica ningún mecanismo de QoS incrementando el tráfico obteniendo valores que no sobrepasan $83.69 \%$ descartando paquetes debido al tiempo de espera. Se puede observar que i-Co ${ }^{2} \mathrm{Q}$ mejora el rendimiento en más de $15 \%$ con respecto a los otros protocolos, incrementando la probabilidad de éxito en la entrega total de paquetes.

La figura $3 \mathrm{~b}$, muestra los resultados obtenidos por la métrica de paquetes perdidos. El promedio total de pérdida de paquetes de los $\mathrm{NC}$ al utilizar i- $\mathrm{Co}^{2} \mathrm{Q}$ es de $4 \%, 2 \%$ y $3 \%$ para las incidencias 1,2 y 3, en comparación con el protocolo [14] que alcanzan hasta el $10 \%$ de paquetes perdidos, transmitidos por nodo ya que cuenta con un tiempo 
de espera mayor. Por otro lado cuando no se utiliza QoS el promedio se eleva a un 57\% de paquetes perdidos conforme se incrementa la transmisión siendo un porcentaje crítico hablando en cuestiones de aplicaciones médicas, con lo cual se observa que i$\mathrm{Co}^{2} \mathrm{Q}$ mejora el rendimiento en alrededor de un $92 \%$.

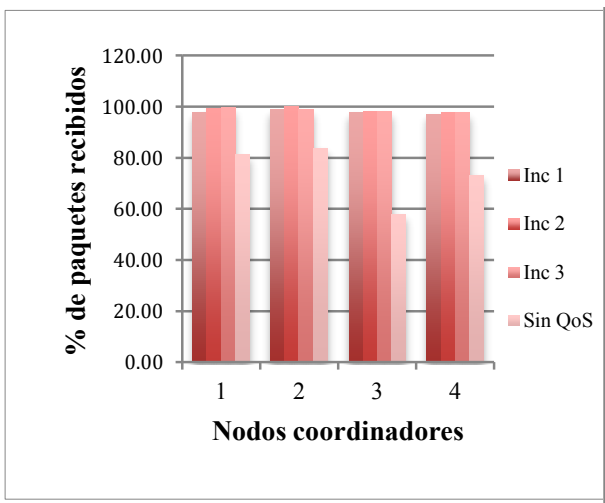

(a)

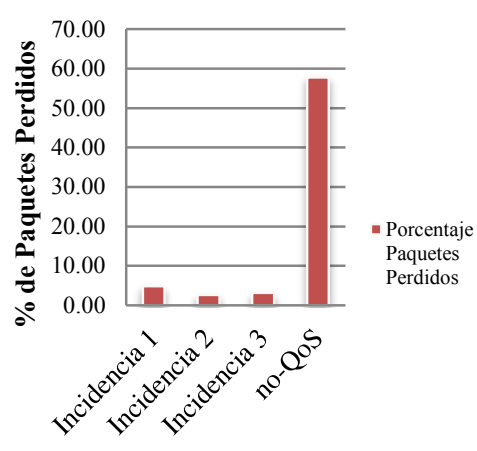

(b)

Fig. 3. Gráficas comparativas con diferentes métricas y diferentes incidencias a) paquetes recibidos por el sink, b) paquetes perdidos.

Finalmente la figura 4 muestra que i-Co ${ }^{2} \mathrm{Q}$ consume alrededor del $1 \%$ más de energía con respecto a no-QoS. En este porcentaje se debe considerar la transmisión exitosa de paquetes y la equidad en el uso de los recursos (fig. 3a) con respecto al consumo de energía por nodo. En el caso de i-Co ${ }^{2} \mathrm{Q}$ es equivalente el consumo de energía a la oportunidad que tiene cada nodo de transmitir sus datos, en comparación con no-QoS que muestra un decremento en la transmisión de datos del nodo 1 al 5.

\section{Consumo de Energía}

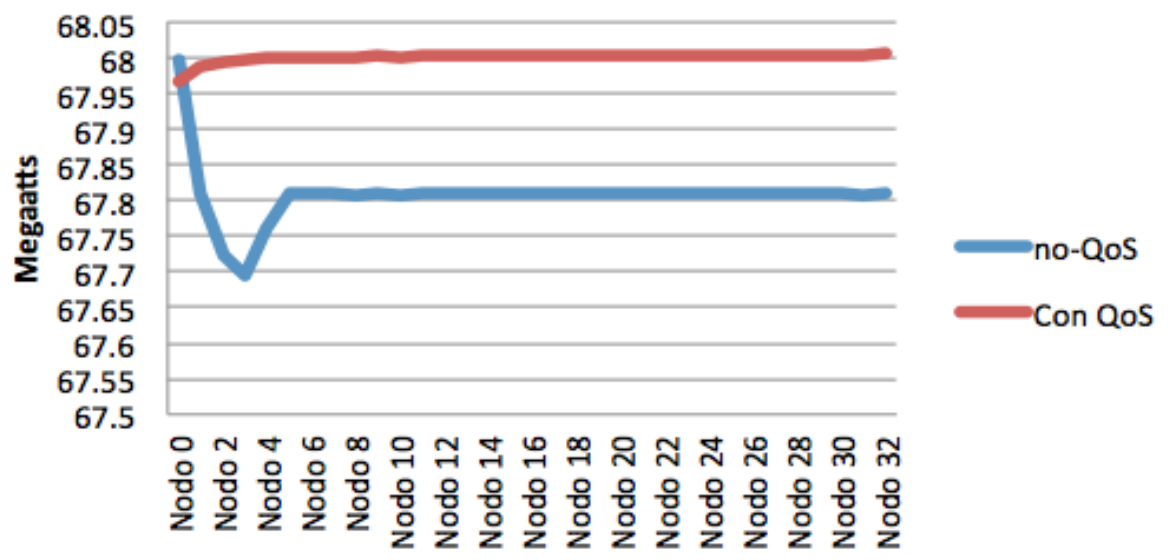

Fig. 4. Consumo de energía. 


\section{Conclusiones}

Si se desea realizar un monitoreo de pacientes en tiempo real de forma eficiente, es necesario implementar protocolos que soporten calidad de servicio para mejorar la eficiencia de transmisión de información heterogéneas dentro de una red WBAN, además de diferenciar los distintos flujos de información y la prioridad de datos.

En este trabajo de investigación se presentó i- $\mathrm{Co}^{2} \mathrm{Q}$ un protocolo inteligente para el control de la congestión haciendo uso de sistema de colas que permita mejorar la eficiencia de la transmisión de los datos heterogéneos con una calidad de servicio en la red.

Los resultados obtenidos nos permiten observar que $\mathrm{i}-\mathrm{Co}^{2} \mathrm{Q}$ es capaz de controlar de forma inteligente la congestión en la red brindando una alta calidad de servicio en redes WBAN.

\section{Referencias}

1. Campaña, B.S., Londoño, P.J.: Estudio de Redes de Sensores y Aplicaciones Orientadas a la Recolección y Análisis de Señales Biomédicas. Colombia (2013)

2. Cervantes, A., Hipólito, J.I., Sánchez, J.D., Martínez, M.E., Calvo, A.: Arquitectura de eSalud basada en redes inalámbricas de sensores (2012)

3. Khan, Z., Sivakumar, S., Phillips, W., Robertson, B.: QPRD: QoS-aware Peering Routing Protocol for Delay Sensitive Data in Hospital Body Area Network Communication, Internetworking Program. Dalhousie University, Canada Saint Mary's University, Canada (2012)

4. León, S.I; Juárez, D.A.: Redes de área corporal en el cuidado de la salud. México, D.F. (2012)

5. Berrahal, S., Boudriga, N.: A Smart QoS- based Traffic Management for WBANs, Communication Networks and Security Research Laboratory. University of Carthage, Tunisia (2014)

6. Buenrostro-Mariscal, R., Nieto-Hipólito, J.I., Cosio-León, M., Yazquez-Briseno, M., Sánchez-López, J.D.: Consideraciones para el Control de congestión en redes inalámbricas de sensores utilizando la optimización crosslayer. Research in Compuring Science 59, pp. 219-230 (2012)

7. Iyer, Y.G., Gandham, S., Venkatesan, S.: STCP: a generic transport layer protocol for wireless sensor networks. In 14th International Conference on Computer Communications and Networks, ICCCN, pp. 449-454 (2005)

8. Shaikh, F.K., Khelil, A., Ali, A., Suri, N.: TRCCIT: Tunable reliability with congestion control for information transport in wireless sensor networks. In The 5th Annual ICST Wireless Internet Conference (WICON), pp. 1-9 (2010)

9. Wan, C.Y., Eisenman, S.B., Campbell, A.T.: CODA: congestion detection and avoidance in sensor networks. In Proceedings of the 1st International conference on Embedded networked sensor systems, SenSys'03, pp. 266-279, New York, NY, USA, ACM (2003)

10. Giancoli, E., Jabour, F., Pedroza, A.: CTCP: reliable transport control protocol for sensor networks. In International Conference on Intelligent Sensors, Sensor Networks and Information Processing, ISSNIP, pp. 493-498 (2008)

11. Castalia. Castalia User Manual. http://castalia.research.nicta.com.au/pdfs/Castalia\%20\%20User\%20Manual.pdf (2011)

12. Varga András. OMNeT++. https://omnetpp.org/

13. Hidalgo, R., Moreno, J.I.: Routing Design in Wireless Sensor Networks and a Solution for Healthcare Environments, IEEE Latin America Transactions (2011) 
i-Co2Q: protocolo inteligente para el control de la congestión consciente de la QoS ...

14. Razzaque, A., Hong, C.S., Lee, S.: Data-centric Multiobjective QoS-aware Routing Protocol for Body Sensor Networks. Sensors, vol. 11, No. 1, pp. 917-937 (2011) 